\title{
A $\mu_{6}$-Oxo Centered Mixed-Valent $\mathrm{Mn}^{\mathrm{III}}{ }_{5} \mathrm{Mn}^{\mathrm{II}}$ Cluster And Its Self-Assembly of An Azido Bridged 1D Chain
}

Kartik C. Mondal, Sauvik Rakshit, Mike G. B. Drew, and Partha S. Mukherjee*

Table-S1: Bond Valence Calculation Table for Complex-2

\begin{tabular}{|c|c|c|c|c|}
\hline $\mathrm{Mn}(\mathrm{m})-\mathrm{O}(\mathrm{n})$ & $\begin{array}{l}\text { Distance }(\overline{\mathrm{A}}) \\
(\mathrm{r})\end{array}$ & $\mathrm{S}_{\mathrm{ij}}$ & $\sum \mathrm{S}_{\mathrm{ij}}$ & Oxidation State \\
\hline $\mathrm{Mn}(1)-\mathrm{O}(47)$ & 2.006 & 0.557 & & \\
\hline $\mathrm{Mn}(1)-\mathrm{O}(77)$ & 2.079 & 0.457 & & \\
\hline $\mathrm{Mn}(1)-\mathrm{O}(71)$ & 2.081 & 0.455 & 3.190 & Mn(Ш) \\
\hline $\operatorname{Mn}(1)-O(45)$ & 2.105 & 0.426 & & \\
\hline $\mathrm{Mn}(1)-\mathrm{O}(2)$ & 1.960 & 0.631 & & \\
\hline $\mathrm{Mn}(1)-\mathrm{N}(121)$ & 1.938 & 0.670 & & \\
\hline $\mathrm{Mn}(2)-\mathrm{O}(2)$ & 2.300 & 0.232 & & \\
\hline $\mathrm{Mn}(2)-\mathrm{O}(61)$ & 1.920 & 0.648 & & \\
\hline $\operatorname{Mn}(2)-O(71)$ & 1.956 & 0.588 & 2.957 & Mn(Ш) \\
\hline $\mathrm{Mn}(2)-\mathrm{O}(65)$ & 1.934 & 0.426 & & \\
\hline $\operatorname{Mn}(2)-O(75)$ & 1.943 & 0.625 & & \\
\hline $\mathrm{Mn}(2)-\mathrm{N}(111)$ & 2.137 & 0.438 & & \\
\hline $\operatorname{Mn}(3)-\mathrm{O}(55)$ & 1.909 & 0.668 & & \\
\hline $\mathrm{Mn}(3)-\mathrm{O}(2)$ & 2.277 & 0.247 & & \\
\hline $\mathrm{Mn}(3)-\mathrm{O}(51)$ & 2.084 & 0.416 & & \\
\hline $\operatorname{Mn}(3)-O(77)$ & 1.921 & 0.647 & 2.997 & Mn(Ш) \\
\hline $\operatorname{Mn}(3)-O(75)$ & 2.085 & 0.415 & & \\
\hline $\mathrm{Mn}(3)-\mathrm{N}(101)$ & 2.018 & 0.604 & & \\
\hline $\mathrm{Mn}(4)-\mathrm{O}(2)$ & 2.387 & 0.199 & & \\
\hline $\mathrm{Mn}(4)-\mathrm{O}(65)$ & 2.117 & 0.413 & & \\
\hline $\mathrm{Mn}(4)-\mathrm{O}(67)$ & 2.084 & 0.451 & 2.369 & Mn(II) \\
\hline $\mathrm{Mn}(4)-\mathrm{O}(55)$ & 2.113 & 0.417 & & \\
\hline Mn(4)-O(57) & 2.124 & 0.405 & & \\
\hline $\operatorname{Mn}(4)-N(133)$ & 2.128 & 0.484 & & \\
\hline
\end{tabular}




\begin{tabular}{|c|c|c|c|c|}
\hline $\mathrm{Mn}(5)-\mathrm{O}(2)$ & 2.278 & 0.246 & \multirow{6}{*}{3.143} & \multirow{6}{*}{ Mn(Ш) } \\
\hline $\mathrm{Mn}(5)-\mathrm{O}(45)$ & 1.929 & 0.633 & & \\
\hline $\operatorname{Mn}(5)-O(41)$ & 1.920 & 0.648 & & \\
\hline $\mathrm{Mn}(5)-\mathrm{O}(51)$ & 1.940 & 0.614 & & \\
\hline $\mathrm{Mn}(5)-\mathrm{O}(57)$ & 1.929 & 0.629 & & \\
\hline $\operatorname{Mn}(5)-\mathrm{N}(131)$ & 2.196 & 0.373 & & \\
\hline $\mathrm{Mn}(6)-\mathrm{O}(2)$ & 2.244 & 0.270 & \multirow{6}{*}{3.039} & \multirow{6}{*}{ Mn(Ш) } \\
\hline $\mathrm{Mn}(6)-\mathrm{O}(61)$ & 2.090 & 0.409 & & \\
\hline $\mathrm{Mn}(6)-\mathrm{O}(67)$ & 1.908 & 0.670 & & \\
\hline $\operatorname{Mn}(6)-O(41)$ & 2.103 & 0.395 & & \\
\hline $\mathrm{Mn}(6)-\mathrm{O}(47)$ & 1.942 & 0.611 & & \\
\hline $\mathrm{Mn}(6)-\mathrm{N}(141)$ & 1.972 & 0.684 & & \\
\hline
\end{tabular}

Reference: Inorg.Chem.1992,31,1585-1588

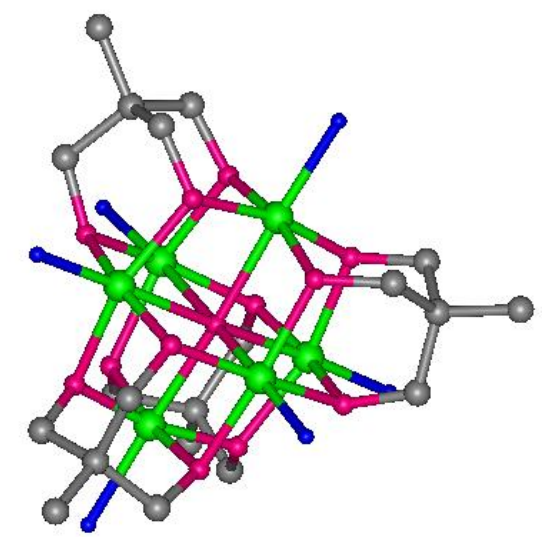

Fig-S1: Basic core unit of the complex-2. 


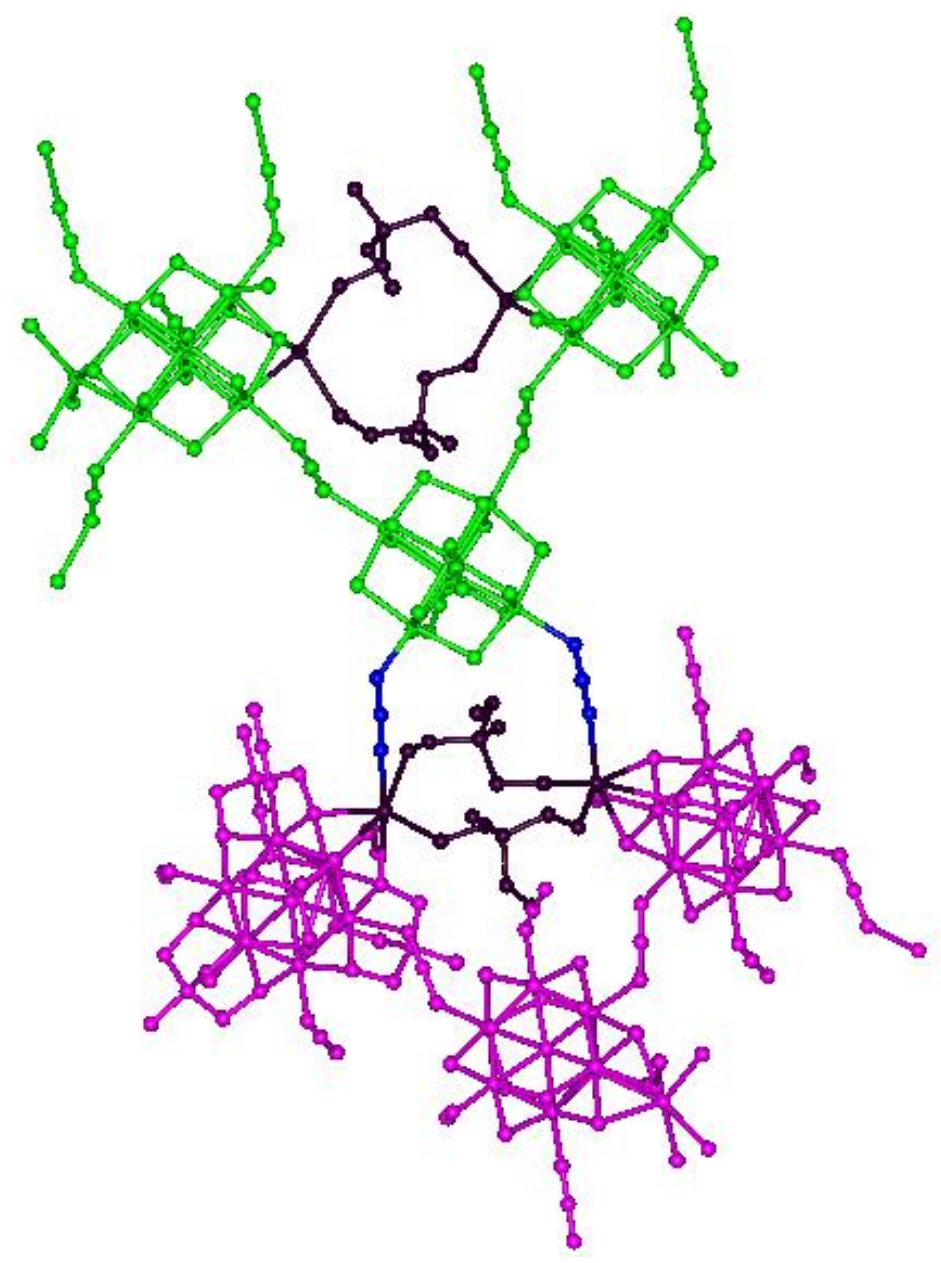

Fig-S2: Represents how the neighboring chains are packed by the counter $\mathrm{Na}^{+}$ions and free $\mathrm{H}_{3}$ thme ligands. Blue $=$ azide; black $=\mathrm{Na}^{+}$and free $\mathrm{H}_{3}$ thme ligands (complex-2). 


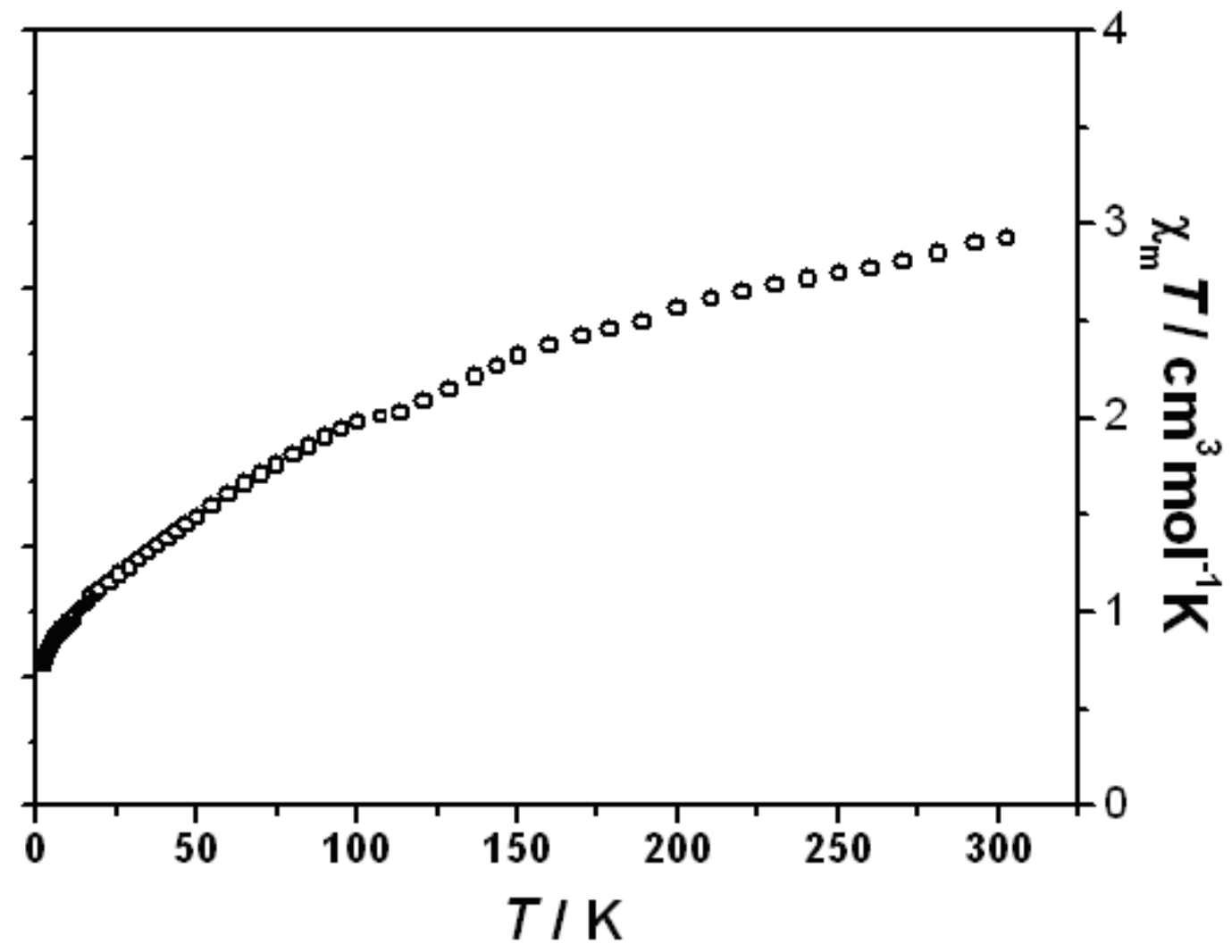

Fig-S3: Plot of $\chi_{M} T$ vs $T$ data for complex-2. 


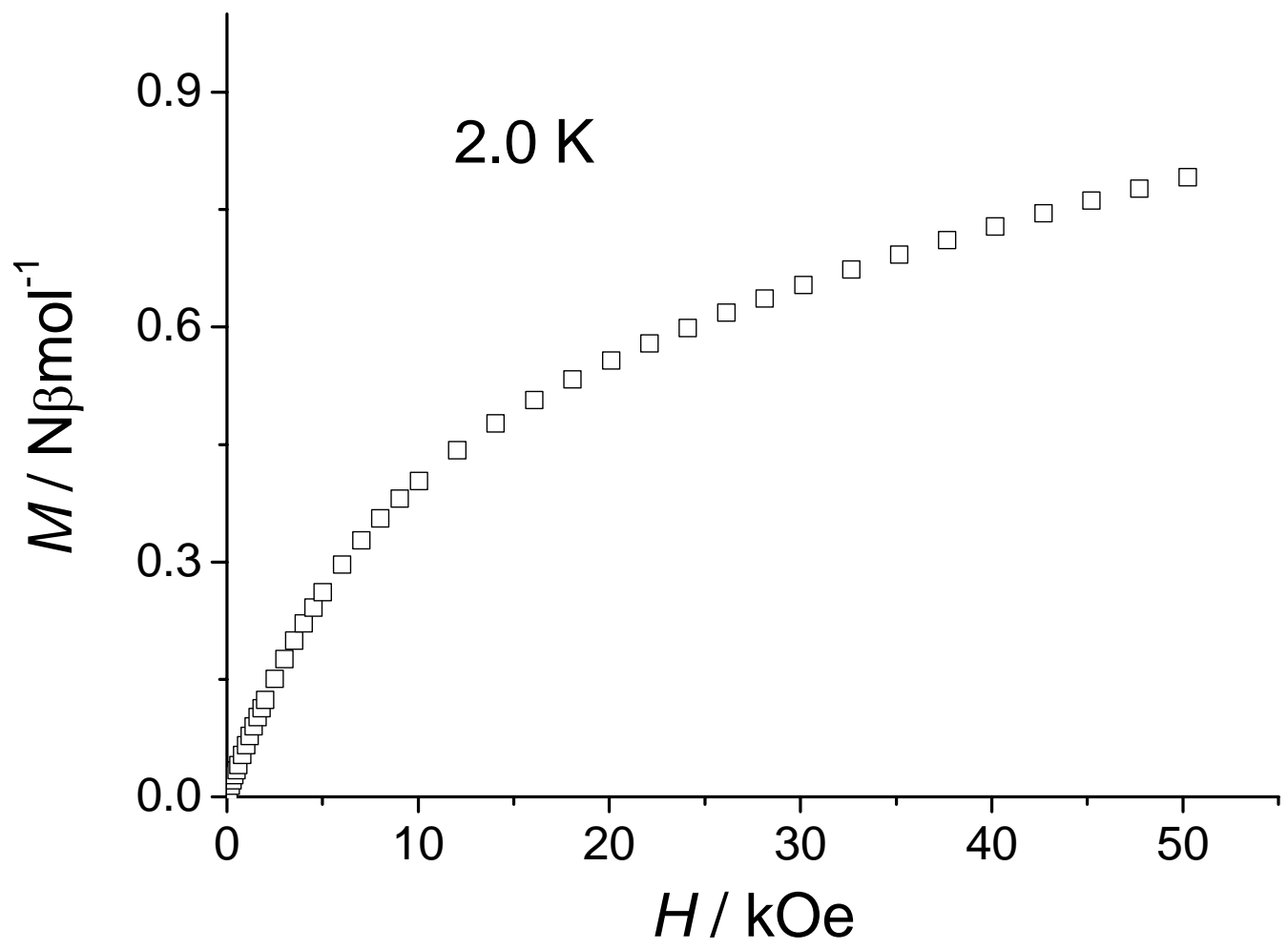

Figure-S4: $\mathrm{M}$ versus $\mathrm{H}$ plot at $2 \mathrm{~K}$ for complex-2. 